\title{
Evaluation of Respiratory Muscles Activity by Means of Cross Mutual Information Function at Different Levels of Ventilatory Effort
}

\author{
Joan Francesc Alonso*, Student Member, IEEE, Miguel A. Mañanas, Member, IEEE, Dirk Hoyer, \\ Zbigniew L. Topor, and Eugene N. Bruce
}

\begin{abstract}
Analysis of respiratory muscles activity is an effective technique for the study of pulmonary diseases such as obstructive sleep apnea syndrome (OSAS). Respiratory diseases, especially those associated with changes in the mechanical properties of the respiratory apparatus, are often associated with disruptions of the normally highly coordinated contractions of respiratory muscles. Due to the complexity of the respiratory control, the assessment of OSAS related dysfunctions by linear methods are not sufficient. Therefore, the objective of this study was the detection of diagnostically relevant nonlinear complex respiratory mechanisms. Two aims of this work were: 1) to assess coordination of respiratory muscles contractions through evaluation of interactions between respiratory signals and myographic signals through nonlinear analysis by means of cross mutual information function (CMIF); 2) to differentiate between functioning of respiratory muscles in patients with OSAS and in normal subjects. Electromyographic (EMG) and mechanomyographic (MMG) signals were recorded from three respiratory muscles: genioglossus, sternomastoid and diaphragm. Inspiratory pressure and flow were also acquired. All signals were measured in eight patients with OSAS and eight healthy subjects during an increased respiratory effort while awake. Several variables were defined and calculated from CMIF in order to describe correlation between signals. The results indicate different nonlinear couplings of respiratory muscles in both populations. This effect is progressively more evident at higher levels of respiratory effort.
\end{abstract}

Index Terms-Electromyography, mechanomyography, muscle activity, mutual information, pulmonary disease.

Manuscript received March 29, 2006; revised January 4, 2007. This work was supported in part by the Comisión Interministerial de Ciencia y Tecnología (CICYT) under contract TEC2004-02274, in part by the Direcció General de Recerca de la Generalitat de Catalunya, Spain, under Contract 1998BEAI200079, in part by the Deutsche Forschungsgemeinschaft (DFG), Germany under contract DFG Ho1634-8-1,2, and in part by the National Institutes of Health (NIH) under Contract HL40369. Asterisk indicates corresponding author.

*J. F. Alonso is with the Department of Automatic Control, Biomedical Engineering Research Center, Technical University of Catalonia (UPC), 5th Pau Gargalo St., E-08028 Barcelona, Spain (e-mail: joan.francesc.alonso@ upc.edu).

M. A. Mañanas is with the Department of Automatic Control, Biomedical Engineering Research Center, Technical University of Catalonia (UPC), E-08028 Barcelona, Spain.

D. Hoyer is with the Biomagnetic Centre, Department of Neurology, Friedrich Schiller University, PF 07737 Jena, Germany.

Z. L. Topor is with the Department of Physiology and Biophysics, Faculty of Medicine, University of Calgary, Calgary, AB T2N 2T9, Canada.

E. N. Bruce is with the Laboratory of Respiratory Dynamics, Center for Biomedical Engineering, University of Kentucky, Lexington, KY 40506-0070 USA.

Digital Object Identifier 10.1109/TBME.2007.893494

\section{NOMENCLATURE}

AAFT Amplitude Adjusted Fourier Transform method.

ANS Autonomic Nervous System.

CMIF Cross Mutual Information Function.

CPAP Continuous Positive Airway Pressure.

DIA Diaphragm muscle.

DIA_EMG Electromyographic signal from diaphragm muscle.

EMG Electromyographic.

FT Fourier Transform.

FT1 Bivariate type of surrogate data based on independent phase randomization of each signal.

FT2 Bivariate type of surrogate data, based on synchronous phase randomization of each signal.

GEN Genioglossus muscle.

GEN_EMG Electromyographic signal from genioglossus muscle.

GEN_MMG Mechanomyographic signal from genioglossus muscle.

HRV Heart Rate Variability.

IP Incremental protocol.

MI Mutual information.

MMG Mechanomyographic.

MMP Maximum Maintained Pressure.

OCMIF Original Cross Mutual Information Function.

OSAS Obstructive Sleep Apnea Syndrome.

SCMIF Surrogate Cross Mutual Information Function.

SMM Sternomastoid muscle.

SMM_EMG Electromyographic signal from sternomastoid muscle.

SMM_MMG Mechanomyographic signal from sternomastoid muscle.

\section{INTRODUCTION}

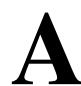
NALYSIS of respiratory muscles activity is an effective tool for the evaluation of pulmonary diseases, such as chronic obstructive pulmonary disease [1], [2] or obstructive 
sleep apnea syndrome (OSAS) [3]. In the model of normal upper airway function, subatmospheric pharyngeal pressures generated by the contraction of the diaphragm must be balanced by opposing forces generated by upper airway dilator muscles such as the genioglossus, in order to prevent a collapse of the upper airways [3]. Sternomastoid is an accessory respiratory muscle activated at high levels of ventilatory effort associated with exercise or with chronic obstructive pulmonary disease [4]. Individuals with OSAS experience intermittent collapse of the upper airways during sleep [3]. Therefore, activity of the respiratory muscles might be expected to differ between OSAS patients and controls, particularly in situations of increased respiratory effort. Patients with OSAS are commonly treated with continuous positive airway pressure (CPAP), which is a pressure introduced through the nasal cavity to provide a pneumatic splint and prevent upper airways from obstructing.

Temporal variations in the activities of respiratory muscles can be assessed from electromyographic (EMG) and mechanomyographic (MMG) signals, which are related to electrical and mechanical muscle activity, respectively. Variables calculated from these myographic signals indicate muscle activity and fatigue during normal and increased respiratory effort [5]-[7]. Temporal interaction between muscles determines the overall muscular pattern during normal and increased respiratory effort. Several interactions have already been assessed by means of different linear techniques such as: cross-spectrum between EMG signals [1] and EMG-MMG signals (to evaluate mechanical-electrical coupling [1], [6]); magnitude squared coherence [8]; and cross-correlation [1]. Although these techniques provide interesting information about a function of different muscles and linear couplings between them, they do not evaluate nonlinear interactions.

Mutual information (MI) analysis represents a general method to detect both linear and nonlinear statistical dependencies between time series and it can be considered as an alternative to the well-known correlation analysis. Cross $\mathrm{Mu}-$ tual Information (CMI) is a statistical function that provides a quantification of both linear and nonlinear components of the coupling between variables and it is based on Shannon's entropy [9]. MI function assesses temporal dependencies in terms of information transfer as a function of time lag. If the amplitude and time resolution is appropriate for the characteristics of the acquired signals, MI is independent of amplitude levels. The only assumption made for data analysis is that measured signals must be realizations of ergodic stochastic processes.

An example of the benefit of nonlinear analysis is provided by the analysis of the complex cardiovascular system behavior. Heart rate variability (HRV) represents the cardiovascular control mediated by the autonomic nervous system (ANS) and central nervous influences, and it is an established and standardized variable in clinical diagnosis. The increasing knowledge of the mechanisms controlling HRV and how they change due to physiological loads and pathophysiological alterations leads to an increasing number of applications of HRV analysis. Since the several cardiac and autonomic mechanisms cannot sufficiently be identified by just one single number, only an approach based on an integrated analysis of both linear and nonlinear heart rate dynamics might contribute to increase the clin- ical sensitivity and specificity [10]. The logical hypothesis that nonlinear complexity analysis would improve upon classical linear analysis, has been partly confirmed both in pathophysiological research and in clinical applications. In that connection, information transfer is a reasonable systems-theoretically founded aspect of functioning of the ANS [11]. CMI analysis has been also used to evaluate coordinations in the cardiorespiratory system [9], [12], [13].

It is not known on which time lags or scales the nonlinear interactions occur. Going beyond linear approaches, measures of complexity, such as correlation dimension, Lyapunov exponents, and Kolmogorov entropy, have improved the assessment of complex autonomic coordination in HRV, for example. Complexity is a well-founded theoretical systems approach to assess complicated systems. All of these complexity measures assess the information transfer independently of time scale. This is an essential limitation with regard to complex multimatched control loops acting on different time horizons within the ANS. Representing interactions on a global time scale, MI carries information on a wider scope of interdependencies (not only "local" but "global" information transfer) than known linear and nonlinear measures described. In this sense, results from [14] confirmed the relevance of global time scale measures of MI for a comprehensive state discrimination in complex systems between HRV and respiratory movement as an example. In our study, the relevant time scales of a measured myographic time series, such as inspiration interval or one (or several) respiratory cycle are not known a priori.

It is known that respiratory rhythm/pattern generation could occur via a nonlinear oscillator [15] or two coupled oscillators [16]. Therefore, the resulting activation of different respiratory muscles could produce complex nonlinear interactions.

In this study, we investigated whether the complex respiratory muscles contractions during breathing could correspondingly be assessed by CMI and whether these contractions were affected by a presence of OSAS. Sternomastoid, and especially genioglossus, which is the most critical muscle in OSAS, play a very important role in the upper airway functionality, but the postulated mechanism by which the activity of these muscles coupled with the activity of the main respiratory muscle (diaphragm) may result in sleep apnea is still unknown. Thus, the purpose of this work was to study couplings between respiratory signals and myographic signals from respiratory muscles through nonlinear analysis using the cross mutual information function (CMIF), and to assess what kind of information could be extracted from this function. These couplings were expected to be different for OSAS patients as compared to healthy controls, since change in respiratory mechanics due to this nocturnal disease might alter muscles properties during wakefulness and therefore muscular and electro-mechanical coupling could change.

\section{Methodology}

\section{A. Signals and Instrumentation}

Eight male patients on CPAP with stable OSAS [age (yr.): $53.8 \pm 10.5$; height $(\mathrm{cm}): 177.0 \pm 8.2$; weight $(\mathrm{kg}): 96.4 \pm 19.2]$ and eight male normal subjects [age (yr.): $45.0 \pm 7.0$; height 
(cm): $177.2 \pm 6.1$; weight $(\mathrm{kg}): 80.5 \pm 10.5$ ] were studied. A Mann-Whitney test with significance level set to 5\%, performed for all anthropometric variables, did not show any statistically significant differences between groups.

Flow signal (F) was acquired with a pneumotacograph (Valydine-CD257). Inspiratory pressure (P) was recorded by means of a strain gauge (COBE) and a carrier demodulator module (Valydine-CD19A). Three surface EMG and two MMG signals were simultaneously recorded from three respiratory muscles: genioglossus (GEN_EMG, GEN_MMG), sternomastoid (SMM_EMG, SMM_MMG) and diaphragm (DIA_EMG). Each EMG signal was recorded with two pre-gelled foam snap $\mathrm{Ag}-\mathrm{AgCl}$ electrodes with a diameter of $8 \mathrm{~mm}$ and with and interelectrode distance of $15 \mathrm{~mm}$. Skin was always cleaned with abrasive paste.

Genioglossus EMG signal was recorded on the submental zone by means of bipolar electrodes [17], [18]. This muscle is innervated by the hypoglossal nerve, CN XII, in an area from 3 to $9 \mathrm{~mm}$ anterior and from 3 to $9 \mathrm{~mm}$ superior to the point where the lingual artery crosses the anterior border of the hyoglossus muscle [19]. This innervation zone was far from the electrode location: in the middle point longitudinally and a perpendicular distance of $2 \mathrm{~cm}$ with respect to a reference line between the chin and the hyoid bone.

The electrodes were placed on the sternomastoid above the innervation zone. The sternomastoid muscle is innervated by the accessory nerve, CN XI. The innervation zone was detected by means of linear electrode arrays below the mastoid process around $35 \%$ of the distance recorded from the sternal notch to the mastoid process [20]. Electrodes were located 20\% of this distance below the mastoid process.

Diaphragm is an inner muscle but activity of its costal fibers can be recorded superficially on the 7th and 8th interspace of the right ventrolateral surface of the rib cage, midway between the clavicular and the axillary lines [8], [21]. Thus, electrodes were placed at this site. As the muscle is innervated internally by phrenic nerve, the recording point was far away from the innervation zone.

The accelerometers (Entran EGA-10) were used to record MMG signals and were placed as closest as possible to the electrodes, in order to record a MMG signal practically at the same point of EMG signal for the posterior analysis of their relationship. Myographic signals were bandpass filtered: $5-40 \mathrm{~Hz}$ (MMG) and 8-400 Hz (EMG). The sampling frequencies were $100 \mathrm{~Hz}$ for MMG and $1000 \mathrm{~Hz}$ for EMG signals.

\section{B. Experimental Protocol}

During the experiment, subjects were awake in supine position breathing through a nose mask connected to a low-resistance nonrebreathing valve. The inspiratory port of the nonrebreathing valve was connected to an external source of a negative pressure. The external pressure was decreased at 90 -second intervals, each time by the value of $-7 \mathrm{~cm} \mathrm{H}_{2} \mathrm{O}$, from zero to the previously determined maximum maintained pressure (MMP) of each subject. The MMP was defined as the magnitude of the lowest external negative pressure below which the subject could no longer generate inspiratory airflow. For every subject, pressure in each step of this incremental protocol (IP) was expressed as a percentage of the individual MMP.

\section{Data Preprocessing and CMIF Calculation}

The flow signal was processed to detect inspiration onsets in order to obtain respiratory rate. EMG and MMG signals were full-wave rectified and demodulated by means of a 400 ms moving average window slid sample by sample [22]. This window produced a low-pass filtering whose magnitude response corresponded to a periodic sinc function where first zero crossing was located at frequency $2.5 \mathrm{~Hz}$ (the inverse of 400 $\mathrm{ms})$. Then resampling was carried out with a final sampling frequency of $20 \mathrm{~Hz}$. Baseline trends of these myographic signals as well as flow and inspiratory pressure were removed to guarantee stationarity. These trends were related to skin-electrode contact, slow motions, and drifts from the instrumentation for flow and pressure recordings among others. The trend removal along the 90-second interval did not affect the existing breathing rhythms and consequently linear or nonlinear couplings between signals during breathing. Then, a transformation of the time series was carried out by ranking data [9], i.e., the transformation of a time series $\{x(t)\} t=1,2 \ldots T$ to a series $\left\{x^{*}(t)\right\} t=1,2 \ldots T$

$$
x(t) \rightarrow x^{*}(t)=T^{-1} \times N\left\{t^{*}: x\left(t^{*}\right)<x(t), t^{*}=1,2, \ldots, T\right\}
$$

where $N$ denotes the number of elements of the set. Ranking data means the substitution of real values by their ranks, that is, changing the lowest and highest values of the signal by 1 and $N$, respectively, and the remaining values assigned to the corresponding intermediate integers. Then, all the values were divided by $N$, so that ranked data $x^{*}(t)$ were always uniformly distributed into the interval $[0,1]$ (unit interval).

The ranking was done to have the best statistics in the entropy estimation; robustness against noise (motion artifacts) and trends was an additional effect [9]. After ranking, any further signal analysis yielded properties that were invariant under monotonic distortions of the signals. Thus, the corresponding series of rank numbers were obtained in each respiratory and myographic signal.

CMIF can be considered as a nonlinear counterpart of the cross-correlation function. The amount of information that was common in both signals was calculated according to the following equation in order to quantify the coupling between two signals $\xi(t)$ and $\eta(t+\tau)$ [13]:

$$
I(\xi, \eta) \equiv H(\eta)-[H(\xi, \eta)-H(\xi)]
$$

where $H(\eta)$ represents the a-priori-uncertainty with regard to $\eta$, and $H((\xi, \eta))-H(\xi)$ is the remaining a-posteriori-uncertainty with regard to $\eta$ if $\xi$ is known. $H$ denotes the Shannon entropy, which is defined as follows [13]:

$$
H(\xi)=-\sum_{a=1}^{A} p_{a} \log _{2}\left(p_{a}\right)
$$


(a) SMM_EMG Signal

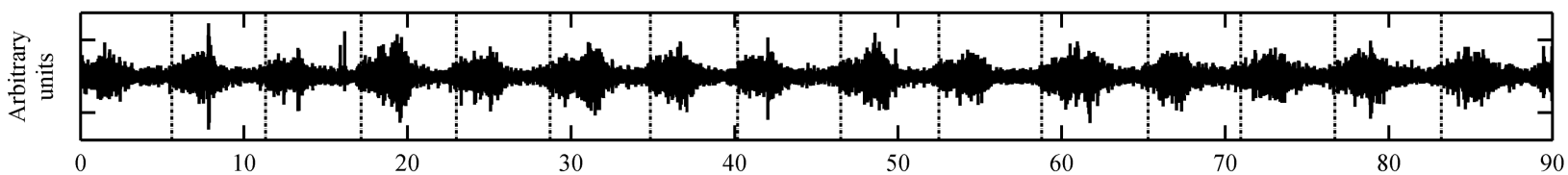

(b) DIA_EMG Signal

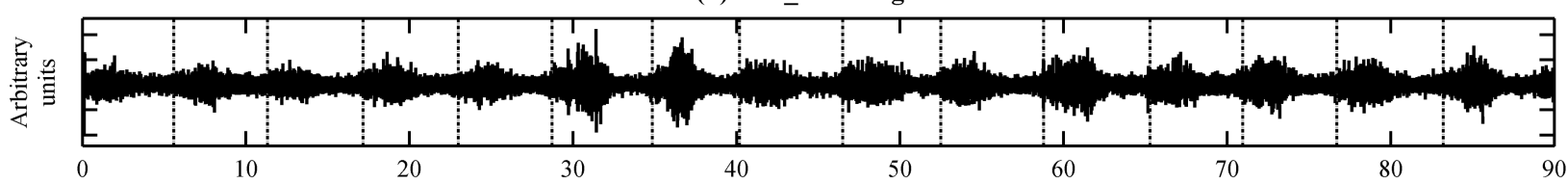

(c) Demodulated SMM_EMG Signal

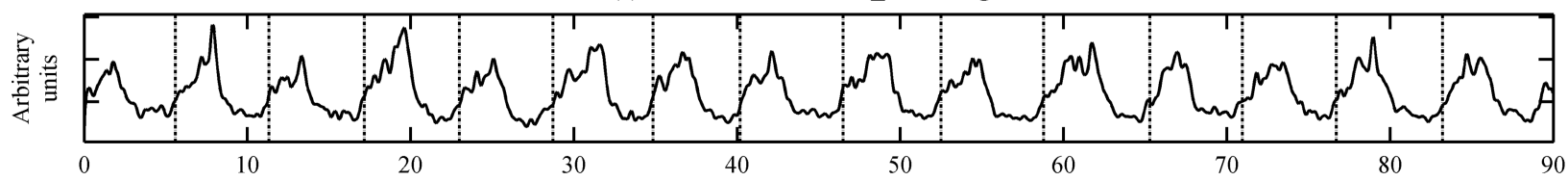

(d) Demodulated DIA_EMG Signal

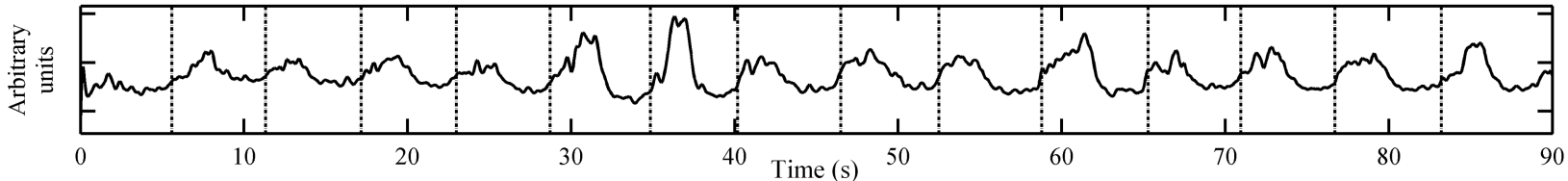

(e) Cross Mutual Information Functions

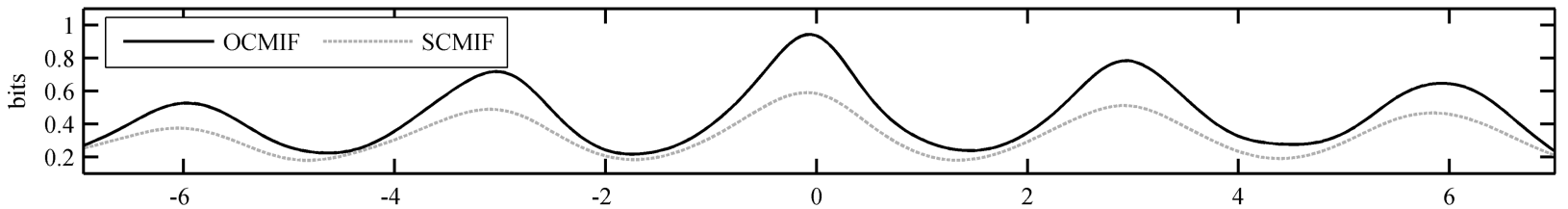

(f) CMIF = OCMIF - SCMIF

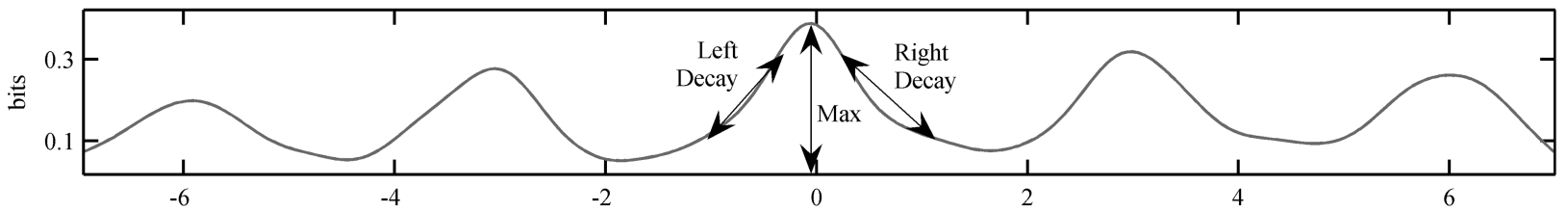

(g) Normalized Cumulative Cross Mutual Information Function

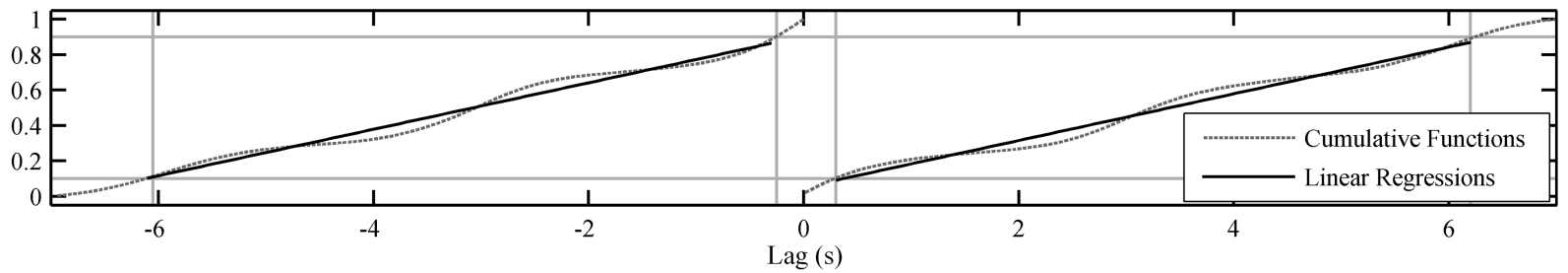

Fig. 1. Examples of myographic signals and CMI functions (40\% of MMP): (a) sternomastoid EMG signal; (b) diaphragm EMG signal; (c) demodulated EMG signal from sternomastoid; (d) demodulated EMG signal from diaphragm; (e) resulting original cross mutual information function (OCMIF) and average of ten FT2 surrogate CMIF (SCMIF); (f) resulting CMIF from the subtraction OCMIF - SCMIF with three variables; (g) normalized cumulative CMIF and linear regression between 0.1 and 0.9 at both sides of the CMIF maximum.

for a discrete distribution $\left\{p_{a}\right\}$ of any random variable $\xi$, when $A$ bins are considered to estimate the histogram $(A=8$ in this study). This histogram is also used as the probability distribution function of the signal.

Finally, CMIF was obtained as a function of time lag $(\tau)$ by repeating the calculation of $I(\xi, \eta)$ for each desired value of $\tau$ ranging from -7 to $7 \mathrm{~s}$. Consequently, CMIF provided complexity measures depending on the time lag and reflecting the information transfer at different time scales. CMIF was calculated in the following pairs of signals: between respiratory signals (P-F); between EMG and MMG signals from the same muscle in order to evaluate electromechanical couplings (GEN_EMG-GEN_MMG and SMM_EMG-SMM_MMG); between MMG signals (GEN_MMG-SMM_MMG) and EMG signals from different muscles (GEN_EMG-SMM_EMG, SMM_EMG-DIA_EMG and GEN_EMG-DIA_EMG) in order to evaluate their coordination. This function calculated from respiratory and myographic signals, which would be modified 


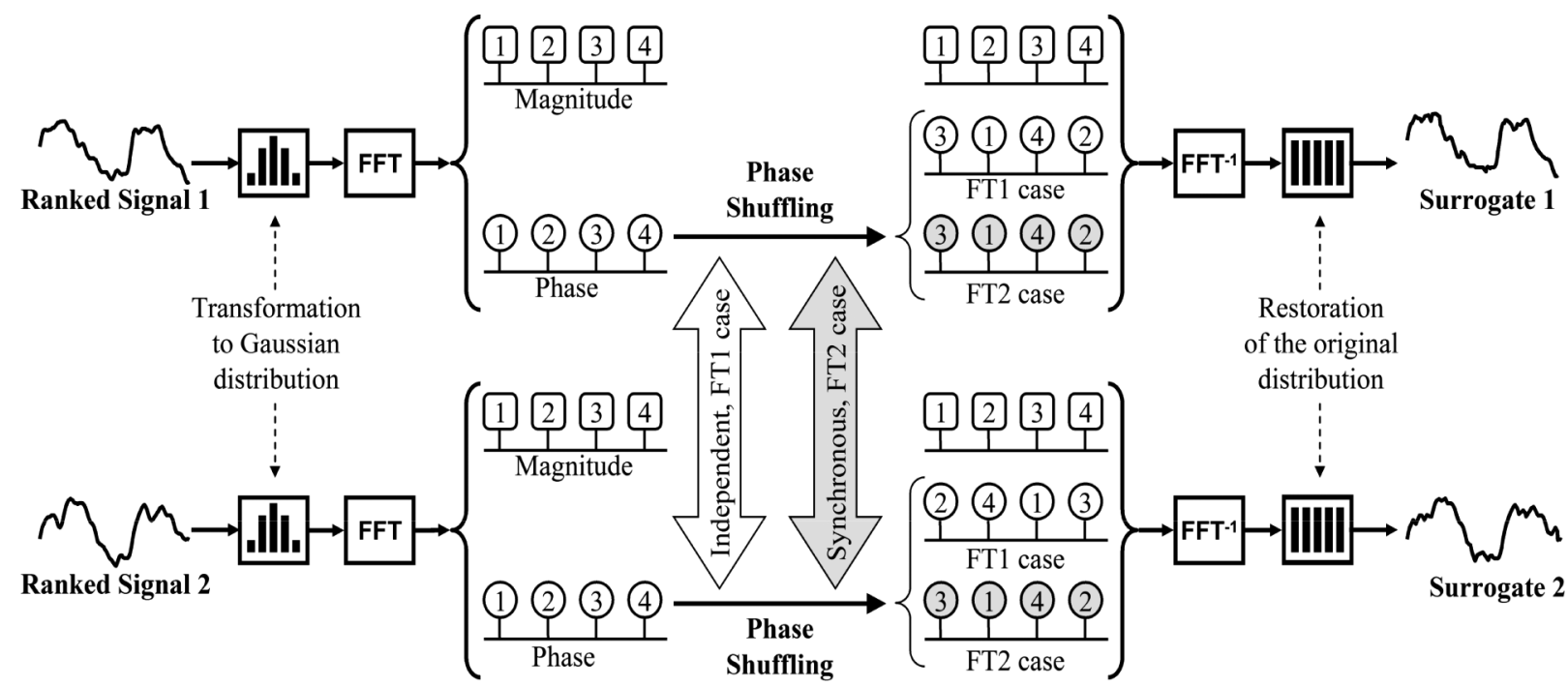

Fig. 2. Calculation procedure for two kinds of surrogate data: FT1 and FT2. In both cases, two surrogates are obtained from two original ranked signals or time series. Difference between FT1 and FT2 cases corresponds to the step of phase shuffling (gray-shaded for FT2).

later by means of surrogate data (see Section II-D), was called original CMIF (OCMIF). Examples of signals and CMIF can be seen in Fig. 1.

\section{Surrogate Data}

Surrogate data come from manipulations of the original data in order to conserve all their statistical properties of the data under study but the property of interest. Special types of bivariate surrogate data were designed based on the amplitude adjusted Fourier transform method (AAFT) in order to identify nonlinear couplings [23]. Surrogate data were obtained by means of the Fourier transform (FT) of the signals: 1) preserving the magnitudes of the FT in order to conserve first and second order statistics, that is, their linear properties; 2) changing randomly the phases of the FT to remove nonlinear properties; and 3 ) transforming back to the time domain.

This method could only be applied to signals with a Gaussian distribution because other kinds of distributions led to spurious nonlinearities [23]. Thus, a transformation of the original data to a Gaussian distribution was carried out previously. After transforming back to the time domain, this transformation was cancelled by restoring the original distribution.

Two types of AAFT surrogates were designed in this study in order to evaluate couplings between two signals (see Fig. 2) [12].

1) FT1 Surrogate Data: Phase randomization was independently applied to each signal. FT1 surrogates corresponded to two independent linear stochastic processes that oscillated with the same frequencies as the original series but asynchronously without any coupling between them;

2) FT2 Surrogate Data: Phase randomization was synchronously applied to both signals. Thus, if there was linear stochastic synchronization between signals, FT2 surrogates would preserve it whereas FT1 would not.

In this study, a surrogate CMIF (SCMIF) was obtained by means of averaging ten CMI functions calculated from surrogate data (either FT1 or FT2) corresponding to each pair of

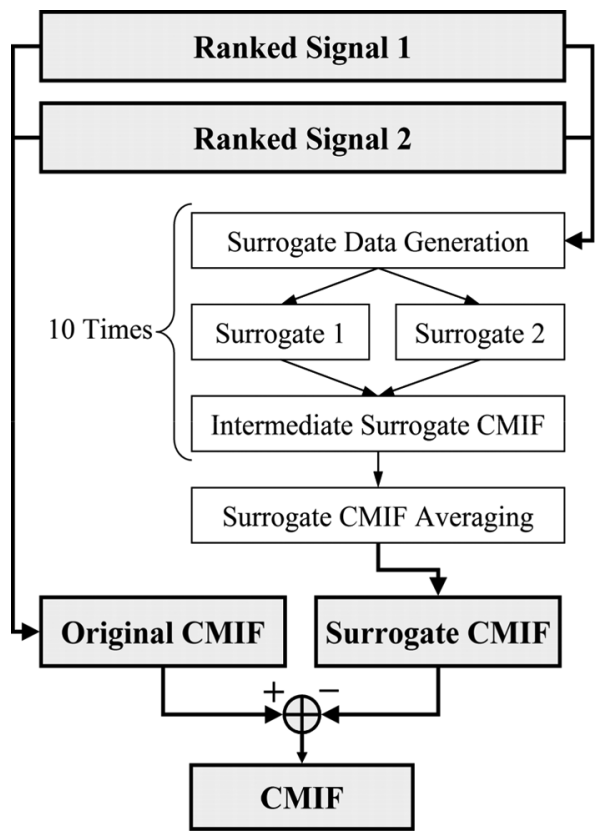

Fig. 3. CMIF calculation procedure after ranking signals (see text). Note that original CMIF is baseline corrected with an averaged surrogate CMIF.

analyzed signals. This SCMIF was subtracted from its corresponding OCMIF and the resulting function of this subtraction was named CMIF, i.e., CMIF = OCMIF - SCMIF. From now on, the terms CMI and CMIF will apply to the result of this subtraction (see Fig. 3). Note that the resulting CMIF from FT1 surrogate subtraction provided information of both linear and nonlinear couplings whereas from FT2 surrogates only nonlinear interactions between signals were considered.

\section{E. Variables of Interest}

Variables were calculated from the CMIF in order to quantify and extract the essential information contained on the function. Generally, a useful variable is the maximum value of the CMIF curve [13], but there are also other fundamental measures like 
the decay of the CMIF from $\tau=0$ to an appropriate close value. Other new variables were defined and calculated from the cumulative CMIF. Thus, the following variables were calculated in this study (see Fig. 1).

1) Maximum Value of the CMIF Around $\tau=0$ (Max): CMIF reached its maximum value near time lag zero because there was no physiologically relevant time shift between signals;

2) Slopes of Main Lobe (Right and Left Decays): CMIF between its maximum and the first local minimum was considered in the direction of higher positive and negative time lags. In both cases, only values from $10 \%$ to $90 \%$ between the maximum and the minimum were considered in order to avoid tail effects. Then, two linear regressions were estimated for positive and negative time lags. Their slopes were called Right and Left Decay, respectively. Decays were considered negative because they were related to the decay of the CMIF from its maximum. Thus, they were related to couplings on short time scale;

3) Slopes from Cumulative CMIF (Right and Left Slopes). Two cumulative functions were obtained integrating CMIF: one function from the lag where maximum of CMIF is located (maxlag) and the second one from the lowest lag considered in the calculation of CMIF which is $-7 \mathrm{~s}$ until maxlag. Both cumulative functions were normalized with respect to their maximum. Then, linear regressions were applied to the values between 0.1 and 0.9 in each cumulative function in order to avoid tail effects. Slopes provided information about the distribution of CMIF along the $7 \mathrm{~s}$, so they were related to couplings on long time scale. However, these $7 \mathrm{~s}$ corresponded to a different number of respiratory cycles depending on the respiratory rhythm of every subject. Slopes from both linear regressions were multiplied by the respiratory period in order to normalize the horizontal axis with respect to this value, and also to know how the information transfer was distributed along relative time (referred as a relative instant during the respiratory cycle) instead of absolute time. After the normalization, both slopes were called Right and Left Slope, respectively.

Statistics were carried out by means of two-sample T-tests in order to compare the two populations. These tests were applied after assuring data normality by means of Kolmogorov-Smirnov tests. T-tests were applied to the average value of the variable at medium and high levels of effort, i.e., from $60 \%$ to $100 \%$ of MMP in each subject. These T-tests were calculated taking into account equal and different variances of data and selecting the correct option according to Levene test [24]. Significance level was set to $5 \%$. Two-sample T-tests were also performed to evaluate differences between the two kinds of surrogate data.

\section{RESULTS}

\section{A. Comparison Between Types of Surrogate Data}

Maximum of CMIF was found near the lag zero. CMIF also showed secondary local maxima at multiples of half respiratory period due to the periodicity of signals. These local maxima appeared especially with respiratory signals, and myographic
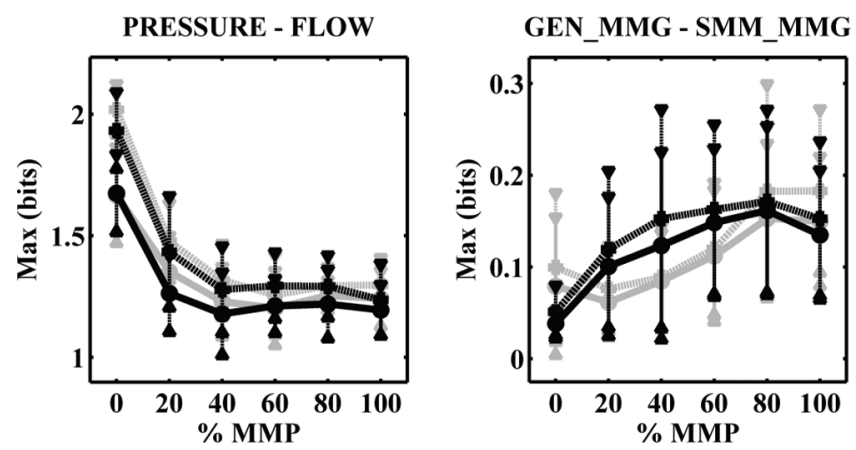

SMM_EMG - SMM_MMG

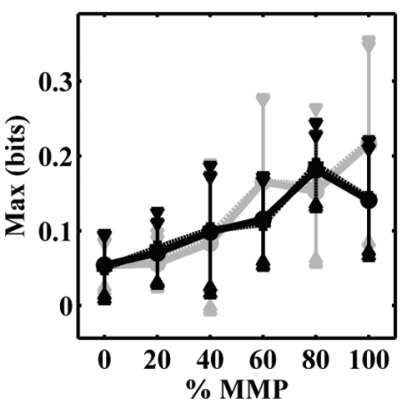

GEN_EMG - SMM_EMG

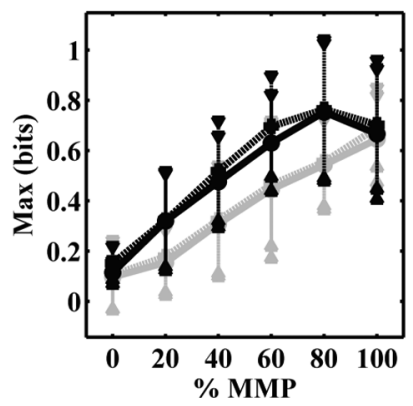

Fig. 4. Max variable from CMIF during the incremental protocol for the two populations (in mean and standard deviation) obtained with two different types of surrogate data (FT1 and FT2). Examples of four types of signal-pairs are shown (P-F, MMG-MMG, EMG-MMG and EMG-EMG). Black traces correspond to patients and gray traces correspond to healthy subjects. Dotted and solid lines correspond to OCMIF-FT1_SCMIF and OCMIF-FT2_SCMIF respectively.

signals at higher ventilatory effort (see Fig. 1, which shows data from one 90-s interval at MMP $=40 \%$ ). This happened in OCMIF as well as with SCMIF from FT1 and FT2 surrogates.

Mean and standard deviation for each population of Max variable with subtracted FT1 and FT2 average surrogates during the incremental protocol (IP) are shown in Fig. 4. Examples of each type of signal-pair can be seen (P-F, MMG-MMG, EMG-MMG and EMG-EMG). Linear regressions were obtained from the variables during the IP in order to evaluate trends by means of one-sample T-tests of their slopes. Max variable showed a clear decrement with higher efforts in the P-F case ( $p$-value $<0.001$ with the slopes from the linear regression during the IP). On the contrary, it clearly increased with the effort in EMG-EMG pairs ( $p$-value $<0.001$ ). A lower increase could also be seen with EMG-MMG (p-value $<0.001)$ and MMG-MMG ( $\mathrm{p}$-value $<0.001$ ) pairs. Concerning myographic signals, variables were very similar using FT1 and FT2 surrogates. Significant differences between the two surrogate subtractions were only found in P-F pair at low levels of effort (p-value $<0.001$ and p-value $<0.05$ at $0 \%$ and $20 \%$ of MMP, respectively). These results were obtained in both populations. Thus, it could be deduced that interaction and coupling between signals were mainly due to nonlinear mechanisms. Results and figures are shown using only FT2 surrogates from now on.

\section{B. Respiratory Information}

Regarding the evolution of Slopes and Decays during the incremental protocol (IP), the difference between populations 

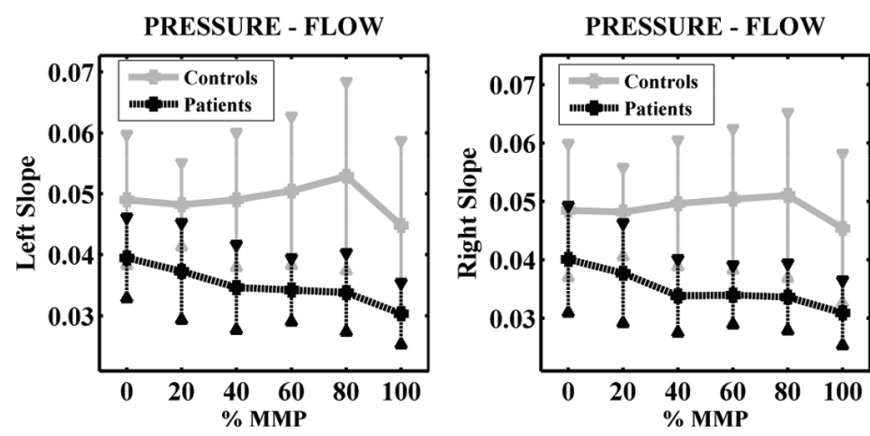

Fig. 5. Variables (see definitions in text) from CMIF between pressure and flow signals. From left to right, Left Slope and Right Slope (p-value < 0.03). Gray solid lines correspond to healthy controls and black dotted lines correspond to OSAS patients in mean and standard deviation of each group.

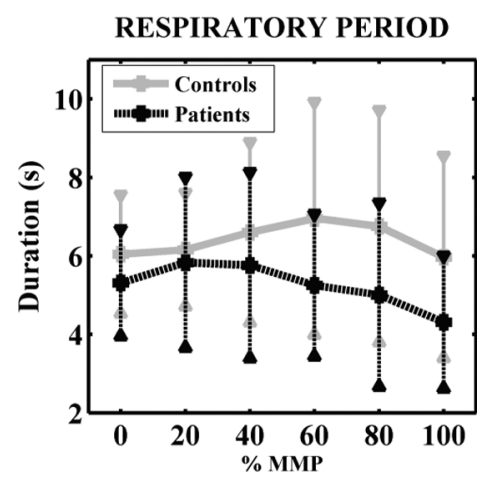

Fig. 6. Respiratory Period during the incremental protocol (IP) as a function of $\%$ MMP. Gray solid lines correspond to healthy controls and black dotted lines correspond to OSAS patients in mean and standard deviation of each group.

generally increased at higher levels of effort. Both types of variables revealed statistically significant differences between the two groups (p-value $<0.05$ for Decays and p-value $<0.03$ for Slopes). Fig. 5 shows the means and standard deviations of Left and Right Slopes of CMIF with P-F signals.

Right and Left Slopes were obtained after the normalization with respect to the respiratory period (Section II-E). In order to verify that the differences between populations were due to a different distribution of information transfer during breathing and not due to a different respiratory rate, the respiratory period was analyzed and statistical differences between groups were not found (Fig. 6).

\section{Mutual Information Between Myographic Signals}

CMIF Slopes with MMG signals between genioglossus and sternomastoid muscles are shown in Fig. 7, in mean and standard deviation for each population. In this case only variables from the cumulative CMIF were different between groups with statistical significance (see Fig. 7).

Fig. 8 shows variables from CMIF between EMG and MMG signals from genioglossus (GEN_EMG-GEN_MMG, upper traces) and sternomastoid (SMM_EMG-SMM_MMG, lower traces) muscles, in mean and standard deviation for each population. Although Right and Left Decays did not show significant differences between populations, Slopes from cumulative CMIF did show them in most cases (see Fig. 8).
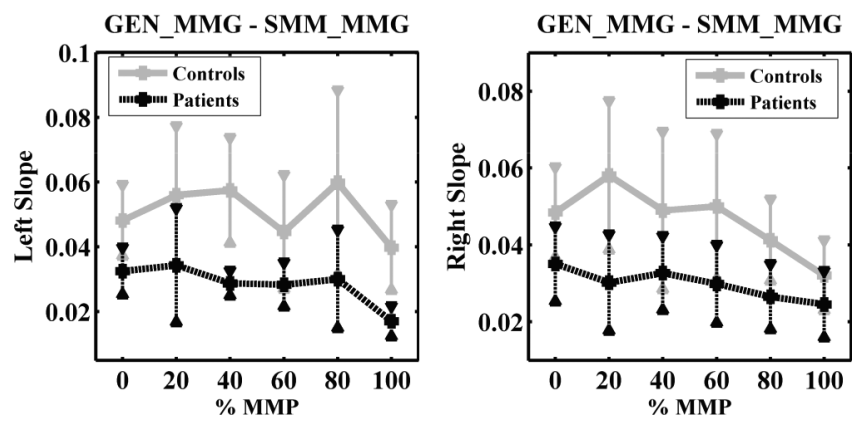

Fig. 7. Variables from CMIF between MMG-MMG signals. From left to right: Left Slope and Right Slope (p-value $<0.02$, p-value $<0.05$, respectively) Gray solid lines correspond to healthy controls and black dotted lines correspond to OSAS patients in mean and standard deviation of each group.
GEN_EMG - GEN_MMG

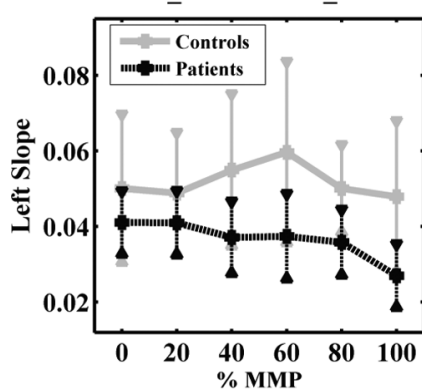

SMM EMG - SMM MMG

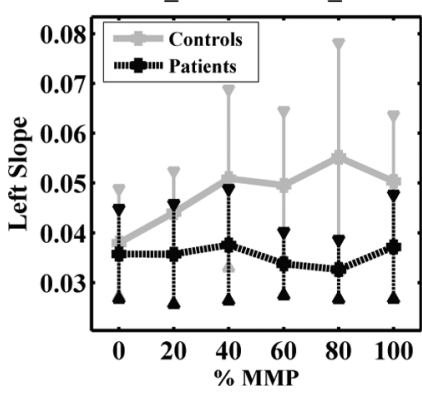

GEN_EMG - GEN_MMG

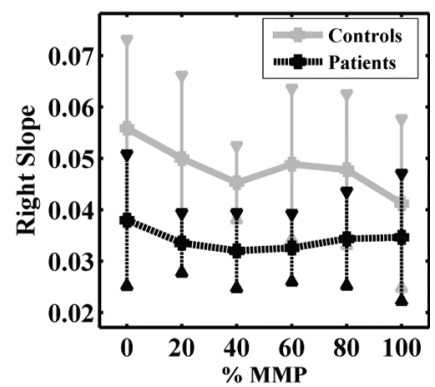

SMM_EMG - SMM_MMG

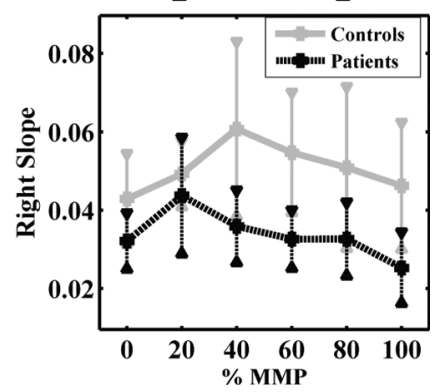

Fig. 8. Variables from CMIF between EMG-MMG signals. Upper traces show Left Slope and Right Slope for genioglossus muscle (p-value $<0.04$, n.s. respectively) and lower traces show the same variables for sternomastoid muscle (p-p - value $<0.05$, value $<0.03$, respectively). Gray solid lines correspond to healthy controls and black dotted lines correspond to OSAS patients in mean and standard deviation of each group.

Fig. 9 shows slopes from CMIF with EMG signals between respiratory muscles [Left and Right Decays, Fig. 9(a); Left and Right Slopes, Fig. 9(b)] in mean and standard deviation for each population. A gradual higher difference between populations was obtained with the increase of effort, especially in Right Decay variable, in all three pairs of EMG signals. Slopes from cumulative CMIF were clearly different between groups at medium and high levels of respiratory effort in any case. Statistics are also shown in Fig 9.

\section{DisCUSSION AND CONCLUSION}

In the last two decades conventional linear methods for biosignal analysis have been substantially extended by nonstationary, nonlinear, and complexity approaches. So far, complexity is usually assessed with regard to one single time 
GEN EMG - SMM EMG

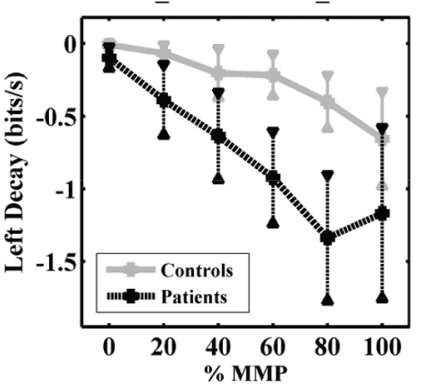

GEN_EMG - DIA_EMG

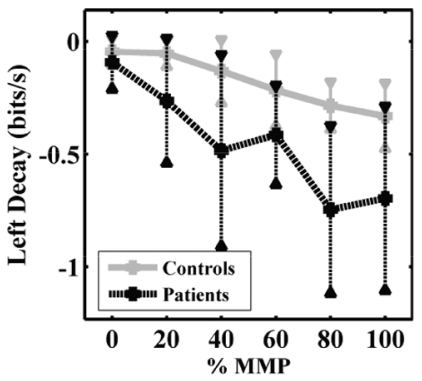

SMM_EMG - DIA_EMG

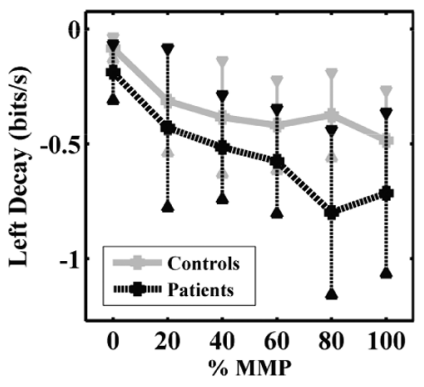

GEN EMG - SMM EMG

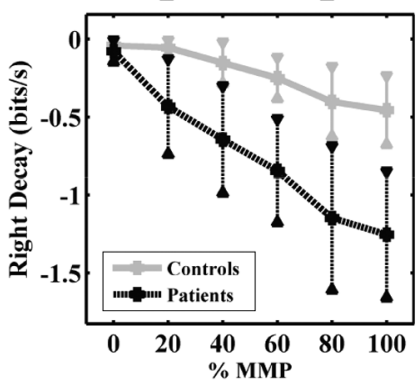

GEN_EMG - DIA_EMG

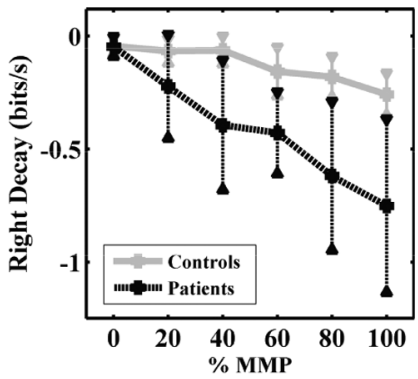

SMM EMG - DIA EMG

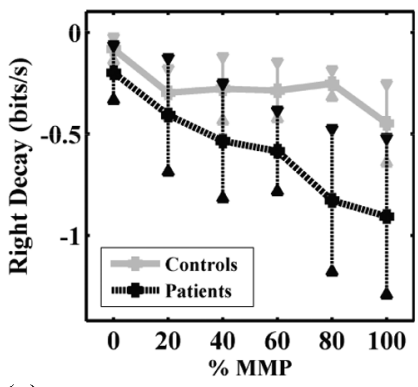

(a)
GEN_EMG - SMM_EMG

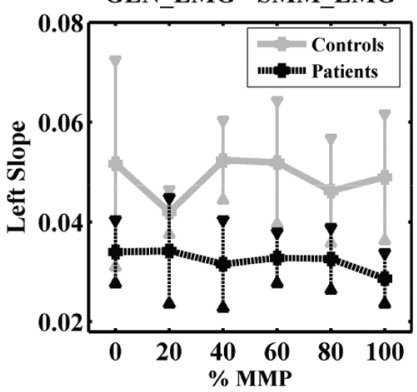

GEN_EMG - DIA_EMG

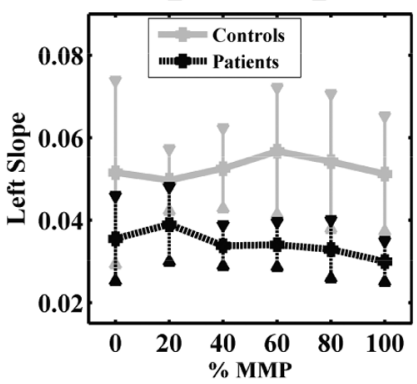

SMM_EMG - DIA_EMG

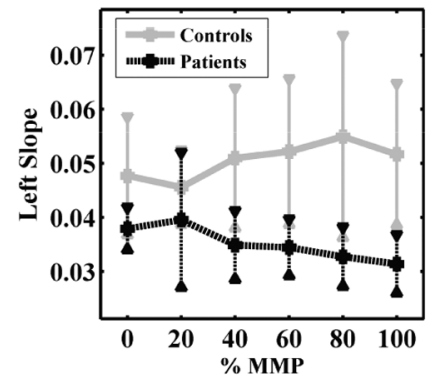

GEN EMG - SMM EMG

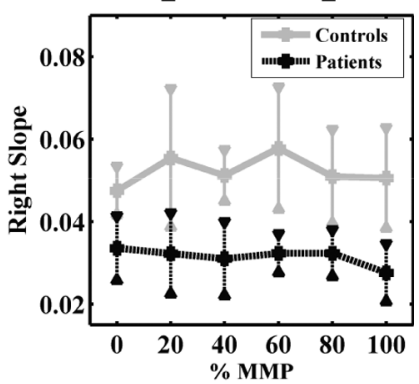

GEN_EMG - DIA_EMG

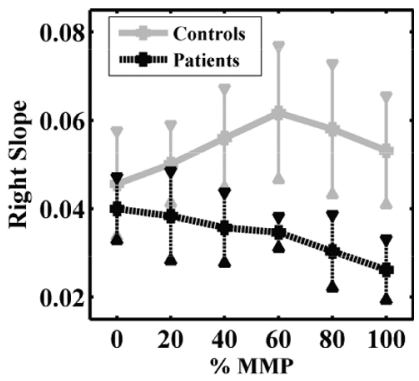

SMM_EMG - DIA_EMG

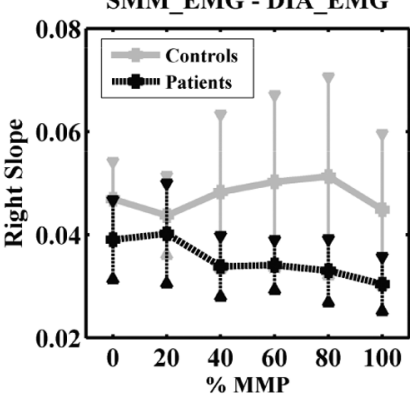

(b)

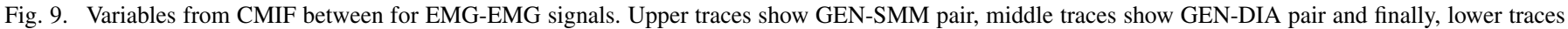

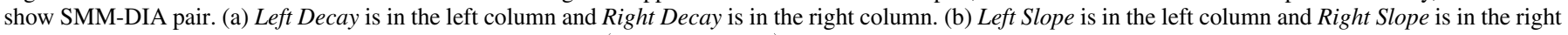

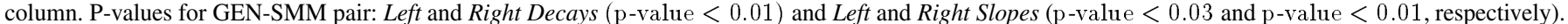

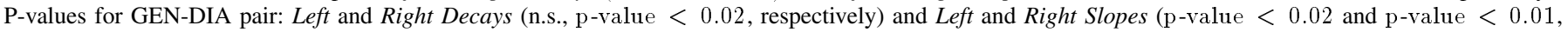

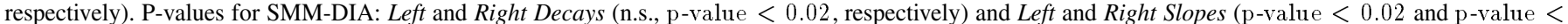

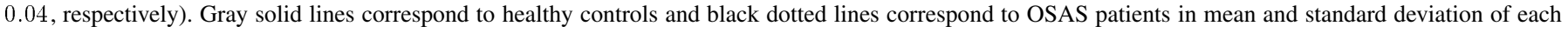
group.

scale, disregarding complex physiology organized on different time scales. This drawback was overcome by information transfer functions. In the present work, CMIF was confirmed as one appropriate possibility of time scale related complexity analysis between myographic signals.

CMI analysis was carried out in order to evaluate interactions between respiratory signals and myographic signals from the respiratory muscles. Evolution of several variables calculated from CMIF during one incremental protocol (IP) provided coupling information. It was deduced that couplings between EMG and MMG signals from the same muscle or between myographic signals from different muscles were basically nonlinear (variables were very similar using FT1 and FT2 surrogates). Thus, techniques which only include linear analysis might fail to detect physiologically significant interactions. Only interaction between respiratory signals presented a linear coupling that was reduced at higher levels of effort. That is because significant differences between FT1 and FT2 surrogates were only found in Max variable until 20\% of MMP. Opposite trends of nonlinear coupling between respiratory signals on the one hand, and between EMG signals on the other hand, were obtained in the Max variable during the IP (increment and decrement, respectively). However, this variable could not differentiate the two subject groups with respect to their muscles and respiratory functions. For this purpose, variables related to the time distribution of information transfer (Decays and Slopes) were considered more suitable compared to absolute values of this information.

It is known that nonlinear interactions between pulmonary and airway afferent activities and integrative central respiratory mechanisms can produce nonrandom (periodic and nonperiodic) variability of the respiratory pattern. Either the respiratory pattern generator can be nonlinear or the disturbance input can be nonlinearly related to the intrinsic variables of the pattern generator [15], [16]. Feedback from airway and muscle mechanoreceptors in humans might be nonlinearly related to 
respiratory effort, and the interactions of the neural oscillator of respiratory pattern with nonlinear feedbacks could cause nonlinear interactions among the outputs of the neural oscillator. Therefore, the resulting activation of different respiratory muscles could produce complex nonlinear interactions.

Respiratory drive to motoneurons is a combination of autonomic (from brainstem) and volitional (from cortex) drives. Cells from the medullary respiratory center in the brainstem have the property of intrinsic periodic firing, and they are responsible for the basic rhythm of ventilation. When all known afferent stimuli have been abolished, inspiratory cells generate repetitive bursts of action potentials that results in nervous impulses going to the diaphragm and other inspiratory muscles [25]. Cortex can override the function of the brainstem within limits. Cortex can activate muscles in parallel and more synchronously (volitional drives could be more related to coupling in long time scales); whereas the brainstem drive acts by way of multiple mechanisms that are temporally synchronized but more independent within the breath (autonomic drives could be associated with couplings in short time scale).

Right and Left Decays showed statistically significant differences between groups in respiratory information and especially with myoelectrical activity between respiratory muscles. They were more negative in OSAS patients than in healthy controls showing in the former a more dramatic decay of CMIF from its maximum value. This maximum was similar in both groups, near the null lag. Reported differences were progressively more evident at higher levels of effort in all the EMG pairs. We can conclude that the loss of nonlinear coordination between respiratory signals and especially in EMG signals recorded from different respiratory muscles is faster in OSAS patients as compared to controls. Thus, short time nonlinear couplings disappeared faster in patients maybe due to afferent activity from the upper airway muscles that modulated the autonomic drive. This effectincreased with higher levels of respiratory effort (higher drives).

Moreover, the best variables in order to detect different behaviors of muscular coordination between groups were Slopes from the cumulative CMIF. Because the used cumulative function was normalized with respect to the total area under the CMIF curve, these slopes considered not only the initial decay of the function from its maximum value but also the remainder nonlinear coupling along the respiratory cycle. In this sense, Right and Left Slopes were lower in OSAS patients especially for EMG signals (p-value $<0.04$ in all pairs), showing that although the first decay was more negative, the nonlinear coordination remained longer than in healthy subjects. This could indicate that cortex had a greater role in patients whose voluntary control of their muscles was more important especially at medium and high levels of respiratory effort. Different respiratory muscles function between the two groups could be detected with these variables at medium and high levels of effort (from $60 \%$ to $100 \%$ of MMP).

In general, results could be interpreted either to suggest that the neural mechanisms had been altered by disease, or that a different balance of (otherwise normal) mechanisms activated the respiratory muscles in OSAS.

The act of breathing requires coordination of multiple muscles. This complex coordination can advantageously be quan- tified by information theoretically based communication measures such as CMIF. Coordination represents the skilful and effective interaction of contractions. The analysis of complex respiratory motor coordination, assessed by information transfer of respiratory muscles, had not been done so far. But only this type of analysis is able to detect nonlinear and complex interrelationships. CMIF is based on joint distributions and it is independent of signal amplitudes. This independence of amplitudes allows a robust assessment of breathing mechanics and neural control information transfer.

Information obtained from CMI analysis provides means to evaluate interaction of respiratory muscles in control and OSAS patients groups as well as to detect differences in their functional coordination. Changes in this complex coordination, such as these associated with OSAS, can quantitatively be characterized by CMIF of myographic signals. Thus, this function and related variables, especially at medium and high levels of effort may provide an effective diagnostic tool. It can be concluded that the respiratory muscles functions are nonlinearly coordinated and that the level of coordination is affected by OSAS.

\section{REFERENCES}

[1] M. A. Mañanas, J. A. Fiz, J. Morera, and P. Caminal, "Analyzing dynamic EMG and VMG signals of respiratory muscles," IEEE Eng. Med. Biol. Mag., vol. 20, no. 6, pp. 125-132, 2001.

[2] M. A. Mañanas, J. F. Alonso, Z. L. Topor, E. N. Bruce, P. Houtz, and P. Caminal, "Frequency parameters from myographic signals for the evaluation of respiratory muscle activity during an increased ventilatory effort," in Proc. IEEE-EMBS, 2003, vol. 4, pp. 3203-3206.

[3] E. Van Lunteren and T. Dick, "Upper airway muscle fiber populations and contractile properties with aging," in Sleep Respiration in Aging Adults, S. T. Kuna, P. M. Suratt, and J. E. Remmers, Eds. : Elsevier, 1991, pp. 229-234.

[4] M. A. Mañanas, R. Jané, J. A. Fiz, J. Morera, and P. Caminal, "Study of myographic signals from sternomastoid muscle in patients with chronic obstructive pulmonary disease," IEEE Trans. Biomed. Eng., vol. 47, no. 5, pp. 674-681, May 2000.

[5] P. Bonato, G. Gaglitati, and M. Knaflitz, "Analysis of myoelectric signals recorded during dynamic contractions," IEEE Eng. Med. Biol. Mag., vol. 15, no. 6, pp. 102-111, 1996.

[6] C. Orizio, "Muscle Sound: Bases for the introduction of a mechanomyographic signal in muscle studies," Crit. Rev. Biomed. Eng., vol. 21, pp. 201-243, 1993.

[7] R. Merletti and L. Conte, "Advances in processing of surface myoelectric signals," Med. Biol. Eng. Comput., vol. 33, pp. 362-372, 1995.

[8] E. N. Bruce and L. M. Akerson, "High-frequency oscillations in human electromyograms during voluntary contractions," J. Neurophysiol., vol. 56, no. 2, pp. 542-553, 1986.

[9] B. Pompe, P. Blidh, D. Hoyer, and M. Eiselt, "Using mutual information to measure coupling in the cardiorespiratory system," IEEE Eng. Med. Biol. Mag., vol. 17, no. 6, pp. 32-39, 1998.

[10] D. Hoyer, H. Friedrich, B. Frank, B. Pompe, R. Baranowski, J. J. Zebrowski, and H. Schmidt, "Autonomic information flow improves prognostic impact of task force HRV monitoring," Comput. Meth. Programs Biomed., vol. 81, no. 3, pp. 246-255, 2006.

[11] D. Hoyer, B. Pompe, K. H. Chon, H. Hardraht, C. Wicher, and U. Zwiener, "Mutual information function assesses autonomic information flow of heart rate dynamics at different time scales," IEEE Trans. Biomed. Eng., vol. 52, no. 4, pp. 584-592, 2005.

[12] M. Palus and D. Hoyer, "Detecting nonlinearity and phase synchronization with surrogate data," IEE Eng. Med. Biol. Mag., vol. 17, no. 6, pp. 40-45, 1998.

[13] D. Hoyer, U. Leder, H. Hoyer, B. Pompe, M. Sommer, and U. Zwiener, "Mutual information and phase dependencies: Measures of reduced nonlinear cardiorespiratory interactions after myocardial infarction," Med. Eng. Phys., vol. 24, pp. 33-43, 2002.

[14] M. G. Frasch, U. Zwiener, D. Hoyer, and M. Eiselt, "Autonomic organization of respirocardial function in healthy human neonates in quiet and active sleep," Early Hum. Dev., Aug. 25, 2006, [Epub ahead of print]. 
[15] E. N. Bruce, "Temporal variations in the pattern of breathing," J. Appl. Physiol., vol. 80, no. 4, pp. 1079-1087, 1996.

[16] K. Vasilakos, R. J. A. Wilson, N. Kimura, and J. E. Remmers, “Ancient gill and lung oscillators may generate the respiratory rhythm of frogs and rats," J. Neurobiol., vol. 62, no. 3, pp. 369-385, 2005.

[17] R. Lydic, L. Wiegand, and D. Wiegand, "Sleep-dependant changes in upper airway muscle function," in Clin. Physiol. Sleep, R. Lydic and J. F. Biebuyck, Eds. Bethesda, MD: American Physiological Society, 1988, pp. 97-123.

[18] S. Duara, M. Rojas, and N. Claure, "Upper airway stability and respiratory muscle activity during inspiratory loading in full-term neonates," J. Appl. Physiol., vol. 77, no. 1, pp. 37-42, 1994.

[19] E. Pumpel and A. Pomaroli, "Point of entrance of the hypoglossal nerve into the genioglossus muscle," Br. J. Oral Maxillofacial Surg., vol. 33 , pp. 391-392, 1995.

[20] D. Falla, P. Dall'Alba, A. Rainoldi, R. Merletti, and G. Jull, "Location of innervation zones of sternocleidomastoid and scalene muscles-A basis for clinical and research electromyography applications," Clin. Neurophysiol., vol. 113, no. 1, pp. 57-63, 2002.

[21] P. W. Hodges and S. C. Gandevia, "Pitfalls of intramuscular electromyographic recordings from the human costal diaphragm," Clin. Neurophysiol., vol. 111, no. 8, pp. 1420-1424, 2000.

[22] E. N. Bruce, "Techniques for electromyography of respiratory muscles," in Tech. Respiratory Physiol., A. B. Otis, Ed. Amsterdam: Excerpta Medica, 1984, pp. 408/1-408/19.

[23] T. Schreiber and A. Schmitz, "Surrogate time series," Physica D, vol. 142, pp. 346-382, 2000.

[24] M. B. Brown and A. B. Forsythe, "Robust tests for the equality of variances," J. Am. Stat. Assoc., vol. 69, pp. 364-367, 1974.

[25] J. B. West, "Control of ventilation," in Respiratory Physiology. Philadelphia, PA: Lippincott Williams \& Wilkins, 1990, pp. 115-130.

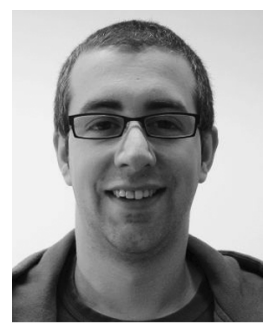

Joan Francesc Alonso (S'02) received the telecommunication engineering degree and the M.S. degree in bioengineering from the Technical University of Catalonia (UPC), Barcelona, Spain, in 2002 and 2004, respectively, where he is working toward the Ph.D. degree.

He is currently working as qualified personnel for investigation support in the Biomedical Engineering Research Center (CREB), UPC, where he takes care of technology transfer tasks. His investigation activity is focused on the interaction analysis between biological signals and systems, with application to EMG, MMG, and EEG signals.
Miguel A. Mañanas (M'99) received the Telecommunication Engineering degree and the $\mathrm{Ph} . \mathrm{D}$. degree in biomedical engineering degree from the Technical University of Catalonia (UPC), Barcelona, Spain, in 1993 and 1999, respectively.

He is currently an Associate Professor and Vice-Director on Research at the Department of Automatic Control and Systems Engineering (ESAII), UPC. He is a member of the Biomedical Engineering Research Center (CREB), UPC, and the Spanish Committee from the International Federation of Automatic Control (CEA). His active research areas include biomedical signal processing, statistical analysis, modeling and simulation. His expertise is specifically in spectral estimation, adaptive algorithms, time-frequency representations, respiratory control system, independent component analysis, and nonlinear techniques applied to EMG, MMG, EEG, and respiratory signals.

Dirk Hoyer received the Diploma of Biomedical Engineering and Information Processing, the Ph.D. degree, and the Doctor of Sciences degree from the Technical University of Ilmenau, Ilmenau, Germany, in 1979, 1985, and 2001, respectively.

From 1992 to 2005, he lead a research group on biosystems analysis at the Institute of Pathophysiology, Friedrich Schiller University, Jena, Germany, where, since 2006, he has been with the Biomagnetic Center, Department of Neurology, and is currently a Lecturer. His research interests are the analysis of complex cardiovascular autonomic control as well as the central nervous information processing. He runs several research projects and clinical studies for risk stratification in cardiac and intensive care patients.

Zbigniew L. Topor received the M.Sc. and Eng. degrees in technical nuclear physics from Stanislaw Staszic Technical University of Mining and Metallurgy, Kracow, Poland, in 1979, and the Ph.D. degree in medical sciences from the University of Calgary, Calgary, AB, Canada, in 1999.

$\mathrm{He}$ is currently working as a Research Associate at the Department of Physiology and Biophysics, University of Calgary. His active research areas include sleep apnea, stability analysis of physiological systems with multiple feedback loops, computer modeling of the respiratory control system. He utilizes computer modeling and system identification techniques to identify underlying pathogenesis of sleep apnea.

Eugene N. Bruce received the Ph.D. degree in biomedical engineering from the University of Southern California, in 1973.

From 2000 to 2003, he was a Program Director with the National Science Foundation (NSF), Arlington, VA. He is currently a Professor in the Center for Biomedical Engineering at the University of Kentucky, Lexington. His current research interests focus on hypoxic brain injury in sleep apnea and carbon monoxide poisoning.

Dr. Bruce is a Fellow of the American Institute for Medical and Biological Engineering (AIMBE), Washington, DC. 